\title{
Ultrasound-guided greater occipital nerve block for patients with occipital headache and short term follow up
}

\author{
Jae Hang Shim, So Young Ko, Mi Rang Bang, Woo Jae Jeon, Sang Yun Cho, Jong Hoon Yeom, \\ Woo Jong Shin, Kyoung Hun Kim, and Jae-Chol Shim
}

Department of Anesthesiology and Pain Medicine, Hanyang University College of Medicine, Seoul, Korea

Background: The greater occipital nerve (GON) block has been frequently used for different types of headache, but performed with rough estimates of anatomic landmarks. Our study presents the values of the anatomic parameters and estimates the effectiveness of the ultrasound-guided GON blockade.

Methods: The GON was detected using ultrasound technique and distance from external occipital protuberance (EOP) to GON, from GON to occipital artery and depth from skin to GON was measured in volunteers. Patients with occipital headache were divided into two groups (ultrasound-guided block: group S, conventional blind block: group B) and GON block was performed. The same parameters were measured on group S and VAS scores were assessed at pretreatment, 1 week and 4 weeks after treatment on both groups.

Results: The GON had distance of $23.1 \pm 3.4 \mathrm{~mm}$ (right) and $20.5 \pm 2.8 \mathrm{~mm}$ (left) from EOP to GON. Its depth below the skin was $6.8 \pm 1.5 \mathrm{~mm}$ (right) and $7.0 \pm 1.3 \mathrm{~mm}$ (left). The distance from GON to occipital artery was $1.5 \pm 0.6 \mathrm{~mm}$ (right) and $1.2 \pm 0.6 \mathrm{~mm}$ (left) in volunteers. Initial VAS score of group S and group B patients were $6.4 \pm 0.2$ and $6.5 \pm$ 0.2 . VAS score of 4 weeks after injection were $2.3 \pm 0.2$ on group $S$ and $3.8 \pm 0.3$ on group $B(P=0.0003)$.

Conclusions: The parameters measured in this study should be useful for GON block and ultrasound-guided blockade is likely to be a more effective technique than blind blockade in occipital headache treatment. (Korean J Anesthesiol 2011; 61: 50-54)

Key Words: Greater occipital nerve block, Occipital headache, Ultrasound, Visual analogue scale.

Received: July 12, 2010. Revised: 1st, August 9, 2010; 2nd, September 9, 2010; 3rd, November 22, 2010; 4th, December 13, 2010. Accepted: December 14, 2010.

Corresponding author: Jae-Chol Shim, M.D., Ph.D., Department of Anesthesiology and Pain Medicine, Hanyang University College of Medicine, 17, Haengdang-dong, Seongdong-gu, Seoul 133-791, Korea. Tel: 82-2-2290-8680, Fax: 82-2-2290-8692, E-mail: jcshim@hanyang.ac.kr (ㄷ) This is an open-access article distributed under the terms of the Creative Commons Attribution Non-Commercial License (http:// creativecommons.org/licenses/by-nc/3.0/), which permits unrestricted non-commercial use, distribution, and reproduction in any medium, provided the original work is properly cited. 


\section{Introduction}

The greater occipital nerve (GON), which derives most of its fibers from the $\mathrm{C} 2$ dorsal root, is the main sensory nerve of the occipital area [1]. The GON is adjacent to the occipital artery and can be located by palpating for this artery.

The indications for the use of the GON injection are not clear. The GON block has been reported to be useful for various headache syndromes such as primary headache [2], cervicogenic headache [3-5], migraine [6,7], occipital neuralgia [8], tension headache [9] and cluster headache [10] and sometimes provides temporary pain relief. Other studies did not mention the effect of the GON block on chronic tension headache [11]. Nevertheless, it has been used for a range of headaches.

Many practitioners still perform the conventional GON block that is based on the anatomical landmarks. Even though realtime ultrasonographic guidance for the GON block is not a popular technique for patients with headache, we hypothesized that the ultrasound guided block is more accurate and safe than the classical blind block.

The aim of this study was to obtain the values of the anatomic parameters and assess the effectiveness of the GON block using ultrasound in the treatment of the occipital headache.

\section{Materials and Methods}

After obtaining approval from the Institutional Review Board and informed consent from the participants, 20 volunteers and 45 patients with occipital headache were enrolled in this study. Forty-five patients had occipital neuralgia and cervicogenic headache, and were divided into two groups. Group S ( $\mathrm{n}=$ 22) was defined as patients receiving the ultrasound-guided GON block and group B $(n=23)$ as patients receiving the conventional blind block. Thirty one subjects had cervicogenic headache and 14 had occipital neuralgia in our study. Sixteen cervicogenic patients and 6 occipital headache patients were included in group S and 15 cervicogenic patients and 8 occipital headache patients in group $B$.

Patients were not allowed to use headache preventive drugs during the study period. Patients who had used acute pain medications within 24 hours prior to the study visit were excluded. Patients with impaired sensation in the occipital area, any neurological or dermatological disease that may have affected skin sensation, bleeding tendency, any skull defect, history of injury to the area of the GON, or any prior adverse reactions to local anesthetics were also excluded.

Healthy volunteers $(n=20)$ included men and women who were, $22-37$ years old. Although we did not inject any solution or needle placement to volunteers, ultrasound measurements were obtained. High-resolution ultrasound imaging was performed using the M-Turbo ${ }^{\mathrm{TM}}$ ultrasound system with a 6-13-MHz high-resolution linear ultrasound transducer (Sonosite, USA). Patients were placed in the prone position with mild neck flexion. After the external occipital protuberance (EOP) was confirmed, an ultrasound probe was placed between the occipital protuberance and the mastoid process parallel to the superior nuchal line. The greater occipital nerves were detected and the distance from the EOP to the GON, from the GON to the occipital artery and the depth from the skin to the GON were measured in the volunteers using ultrasound (Fig. 1). Three of a total of 20 cases on the right side and 2 of a total of 20 cases on the left side were excluded due to invisibility.

For local anesthetic injections for group S and group B patients, patients were placed in the same position as volunteers and the affected side of the GON was detected. Patients in group $\mathrm{S}$ had the same measurements taken as the volunteers using ultrasound (Fig. 1). For the classical infiltration technique used in group B, the parameters previously mentioned in this preliminary study were used. We palpated the EOP and a needle was inserted between $15 \mathrm{~mm}$ and $25 \mathrm{~mm}$ lateral to the EOP just above the superior nuchal line of the affected side. If we could detect palpation of the occipital artery, local anesthetic was injected around the artery after aspiration to prevent
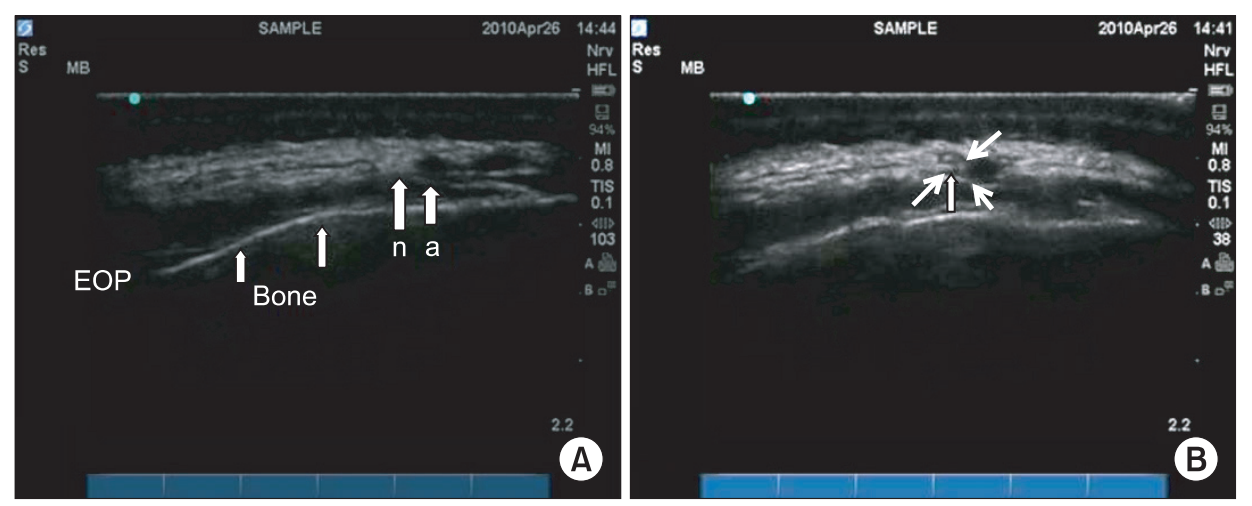

Fig. 1. Ultrasound image of the greater occipital nerve (GON) and the occipital artery (a) of the left side away from the midline (external occipital protuberance; EOP) is seen (A). Fluid accumulation of local anesthetic (thin arrow) is seen around the greater occipital nerve (thick arrows) after injection (B). 
intravascular injection based on the preliminary study.

A $3 \mathrm{ml}$ syringe containing a total of $1 \mathrm{ml}$ of $0.1 \%$ chirocaine (Abott, USA) and $2 \mathrm{mg}$ dexamethasone (Yuhan, Korea) was prepared for each nerve injection for both groups. Patients were given a unilateral or bilateral GON block using a 25-gauge needle. The side effects of injection were determined by clinical symptoms.

For both groups, we evaluated the success of the block 15 minutes after the injection of medication. We checked for the sensory changes such as numbness or sensory loss of the dermatome. If there were no sensory changes, we decided that the GON block was failed. Three patients failed in group B, so they were excluded from data analysis.

Demographic data was collected. Headache severity was assessed on a visual analogue scale (VAS) scored in a blinded manner. Patients were assessed prior to being injected and 1 week and 4 weeks after treatment.

Statistical analyses were performed using StatView (SAS Institute Inc., version 5.01, USA). Data are presented as the mean \pm SD and the demographic data were analyzed using the unpaired t-test and one-way analysis of variance. The diffe-

Table 1. Demographic Data of Two Groups and Volunteers

\begin{tabular}{lccc}
\hline & Group S & Group B & Volunteers \\
\hline Age (yr) & $58.5 \pm 11.7$ & $54.4 \pm 10.4$ & $30.1 \pm 4.2$ \\
Gender (M/F) & $10 / 12$ & $9 / 11$ & $6 / 14$ \\
Height (cm) & $161.7 \pm 9.5$ & $160.8 \pm 10.0$ & $164.0 \pm 7.0$ \\
Weight (kg) & $61.6 \pm 9.8$ & $61.0 \pm 8.6$ & $60.0 \pm 9.0$ \\
Affected site (R/L) & $10 / 13^{*}$ & $10 / 10$ & $17 / 18$ \\
\hline
\end{tabular}

The values are reported as the mean \pm SD. Group S $(n=22)$ defined as patients who received the ultrasound-guided greater occipital nerve (GON) block and group $B(n=20)$ as patients who received conventional blind block. R: right, L: left. *One patient of Group S had a bilateral GON block.

Table 2. Anatomic Parameters of Volunteers and Group S Patients

\begin{tabular}{lcrrrr}
\hline & \multicolumn{2}{c}{ Volunteers } & & \multicolumn{2}{c}{ Group S } \\
\cline { 2 - 3 } \cline { 5 - 6 } & Right $(\mathrm{mm})$ & Left $(\mathrm{mm})$ & & Right $(\mathrm{mm})$ & Left $(\mathrm{mm})$ \\
\hline Depth & $6.8 \pm 1.5$ & $7.0 \pm 1.3$ & & $6.8 \pm 1.1$ & $7.3 \pm 1.0$ \\
EOP to GON & $23.1 \pm 3.4$ & $20.5 \pm 2.8$ & & $22.1 \pm 5.0$ & $19.9 \pm 3.0$ \\
GON to OA & $1.5 \pm 0.6$ & $1.2 \pm 0.6$ & & $1.3 \pm 0.5$ & $1.7 \pm 0.5$ \\
Size of GON & $2.9 \pm 0.4$ & $3.1 \pm 0.3$ & & $3.0 \pm 0.3$ & $3.0 \pm 0.2$ \\
\hline
\end{tabular}

The values are reported as the mean $\pm \mathrm{SD}$. Depth: from the skin to the greater occipital nerve (GON), EOP to GON: distance from the external occipital protuberance to the GON, GON to OA: distance from the GON to the occipital artery. Measurement was possible in 17 of 20 caseson the right and 18 of 20 cases on the left in the volunteers: In three cases on the right and two cases on the left, the greater occipital nerve was not clearly identifiable by ultrasound. Measurement was possible in 10 of 12 cases on the right and 13 of 13 cases on the left in group S: In two cases on the right, the greater occipital nerve was not clearly identifiable by ultrasound. rences in VAS score at pretreatment, 1 week after injection and 4 weeks after injection were analyzed using the unpaired t-test. A $P$ value of $<0.05$ was considered statistically significant.

\section{Results}

There were no significant differences between the two groups (group S and group B) in age, gender, weight, and height (Table 1).

Twenty volunteers were enrolled for the preliminary study and each parameter was collected. The greater occipital nerve had a distance of $23.1 \pm 3.4 \mathrm{~mm}$ on the right and $20.5 \pm 2.8 \mathrm{~mm}$ on the left (range: right 16.6-27.9 mm; left 16.7-25.3 mm) from the EOP to the GON. The depth from the skin was $6.8 \pm 1.5 \mathrm{~mm}$ on the right and $7.0 \pm 1.3 \mathrm{~mm}$ on the left (range: right $5.0-10.1$ $\mathrm{mm}$; left 4.8-9.8 $\mathrm{mm}$ ). The distance between the GON and the occipital artery was $1.5 \pm 0.6 \mathrm{~mm}$ on the right and $1.2 \pm 0.6 \mathrm{~mm}$ on the left (right: range $0.0-2.0 \mathrm{~mm}$; left: range $0.4-2.1 \mathrm{~mm}$ ) (Table 2).

Twelve women and 10 men were enrolled for the ultrasoundguided GON block (group S). The greater occipital nerves were successfully visualized in 10 of the 12 cases on the right and 13 of the 13 cases on the left. The results of parameters showed no significant differences compared with volunteers (Table 2).

The initial VAS score of patients in group $\mathrm{S}$ and group B were $6.4 \pm 0.2$ and $6.5 \pm 0.2$, respectively. There was no statistical difference between groups. The VAS score of 1 week after treatment was $2.3 \pm 0.2$ for group $S$ and $3.0 \pm 0.3$ for group $B$, constituting no statistical difference $(\mathrm{P}=0.068)$. The VAS score of 4 weeks after treatment was $2.3 \pm 0.2$ for group $S$ and $3.8 \pm 0.3$

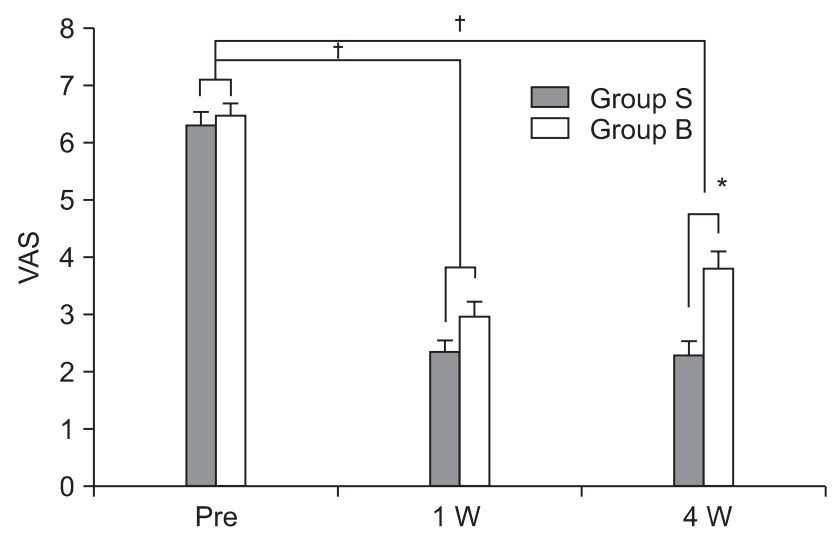

Fig. 2. Comparison of VAS score between the two groups on each time course is seen. Group $S(n=22)$ defined as patients with ultrasound-guided greater occipital nerve (GON) block and group $B(n=20)$ as patients with conventional blind block. Two patients were not reached to obtain the VAS score at 4 weeks for group $S$ and so dropped off. $* \mathrm{P}<0.05$ compared with group $\mathrm{B}$ at 4 weeks after treatment. ${ }^{\dagger} \mathrm{P}<0.05$ compared with pretreatment on both groups. Pre: before greater occipital nerve block, $1 \mathrm{~W}$ : 1 week after block, $4 \mathrm{~W}$ : 4 weeks after block. 
for group $\mathrm{B}$, which is a statistically significant difference $(\mathrm{P}=$ 0.0003). There were significant differences $(P<0.05)$ between the initial VAS score and the VAS score of 1 week and 4 weeks after treatment on each group (Fig. 2).

Adverse effects such as vaso-vagal syncopal attack, transient dizziness following the injection, alopecia around the injection site and exaggerated headache were not reported.

\section{Discussion}

Peripheral nerve blocks have long been used in headache treatment. The most widely used procedure for this purpose has been the greater occipital nerve block. Several studies have suggested the GON block as an effective treatment for various kinds of headache. The rationale for using a GON block in headache treatment comes from evidence of the convergence of sensory input to the trigeminal nucleus caudalis neurons from both cervical and trigeminal fibers $[6,12]$, and its role in antagonizing a putative "wind-up-like effect", which may explain the headache improvement [4].

The two conditions commonly treated with GON blocks are occipital neuralgia and cervicogenic headache. Occipital neuralgia and cervicogenic headache are defined by the International Classification of Headache Disorders, $2^{\text {nd }}$ Edition [13]. Cervicogenic headache (CGH) is a chronic hemicranial pain usually beginning in the suboccipital region and spreading anteriorly to the ipsilateral orbital, frontal, and temporal areas. This headache, of almost daily occurrence, is typically dominant on one side, but may occasionally be bilateral [14]. Headaches were significantly relieved 1 week and 4 weeks after injections when compared with pretreatment.

Although there is no standardized procedure for the GON blockade, the nerve is usually infiltrated with a mixture of a local anesthetic and corticosteroid under blind technique. Ambrosini et al. [10] mentioned that local anesthetics with steroid was more effective than local anesthetic alone for suboccipital injection. Therefore we used a mixture of $1 \mathrm{ml}$ of chirocaine $0.1 \%$ and $2 \mathrm{mg}$ dexamethasone. We used a small amount of local anesthetic in comparison to other studies $[3,15,16]$. Three cases in group B were not properly blocked. The volume of the injection may not be enough to create a block. A small volume may be enough for a greater occipital nerve blockade under ultrasound guidance.

The unusual adverse effects of a GON block, including Cushing's syndrome [15] , vaso-vagal syncopal attack, transient dizziness following the injection, alopecia around the injection site and exaggerated headache [17], were not observed in our study.

The greater occipital nerve is the medial branch of the posterior division of the second cervical nerve, and traverses the posterior scalp and neck. Ducic et al. [18] reported that the GON proceeds between the inferior oblique and the semispinalis capitis muscle and then pierces medially to the semispinalis capitis muscle. It courses obliquely, in a superolateral fashion and is considerably more lateral at the level of the occipital prominence. It then penetrates through the trapezius muscle to join the occipital artery. Their study suggested that individual treatment failures of blind nerve blocks for the treatment of occipital headaches could have occurred because the patients might have had anatomical variants such that the nerve block did not hit the nerve. Therefore the ultrasound-guided blockade might be a useful technique for increasing the success rate of the GON block.

Schmidt and Adelmann [19] mentioned that the occipital artery followed a tortuous course, including an occasional hairpin bend. The occipital artery runs at a mean distance of $3.92 \mathrm{~cm}$ on the right side and $4.4 \mathrm{~cm}$ on the left side from the midline of the external occipital protuberance of the occiput. A comparison between the right and left side showed a marked side-difference in the course of the vessel and the extent of tortuosity varied distinctly. Greher et al. [20] reported that the distance from the EOP to the GON was $17.4 \mathrm{~mm}$ (11.1$22.8 \mathrm{~mm}$ ) in their cadaver study. In our study, the distance between the GON and the occipital artery was $1.5 \pm 0.6 \mathrm{~mm}$ on the right and $1.2 \pm 0.6 \mathrm{~mm}$ on the left, representing no significant difference. The GON was a distance of $23.1 \pm 3.4 \mathrm{~mm}$ on the right and $20.5 \pm 2.8 \mathrm{~mm}$ on the left from the EOP to the GON. Our results were similar to Greher's results. The average diameter of the GON measured by ultrasound, was $2.9 \pm 0.4$ $\mathrm{mm}$ on the right and $3.1 \pm 0.3 \mathrm{~mm}$ on the left in our study. The results are similar to past studies $[18,21,22]$.

Because the greater occipital nerve enters the subcutis below the external protuberance of the occiput and shortly afterwards crosses the occipital artery, many practitioners palpate this artery to detect the occipital nerve while using the conventional blind block. Yet there was considerable interpatient variability in the nerve location. The use of the ultrasound guided block improved accuracy in locating the occipital nerve and did not require patient cooperation for optimal detection of the nerve compared to other techniques [3].

One important factor that might have contributed to the better VAS score of group $S$ is the ability to detect and block the GON precisely rather than simply infiltrating its general location using a field block technique. Accurate injection is also important diagnostically as false localization may lead to unnecessary surgical transection or other neurolytic procedures [23]. Hence, the ultrasound guided technique enables the operator to determine the exact location of the nerve, increasing chances of success with small quantities of the drug.

A major limitation of this study is the short duration of followup. The follow-up assessment was done at 1 week and 4 weeks, 
based on our clinical experience with the duration of GON block effects. A longer follow-up may have been necessary to detect significant group differences of outcome measures. Nevertheless, this study is, we believe, the first ultrasoundguided block of the greater occipital nerve for live patients and our measured parameters in this study should be useful for the greater occipital nerve block.

In conclusion, an ultrasound-guided occipital nerve blockade is a treatment that provides relief from occipital headache and its accompanying symptoms for up to four weeks. This simple and effective technique merits further investigation for patients suffering from occipital headache.

\section{References}

1. Bogduk N. The clinical anatomy of the cervical dorsal rami. Spine (Phila Pa 1976) 1982; 7: 319-30.

2. Lim HB, Hunt K. Anesthetic management for surgical placement of greater occipital nerve stimulators in the treatment of primary headache disorders. J Neurosurg Anesthesiol 2007; 19: 120-4.

3. Naja ZM, El-Rajab M, Al-Tannir MA, Ziade FM, Tawfik OM. Occipital nerve blockade for cervicogenic headache: a doubleblind randomized controlled clinical trial. Pain Pract 2006; 6: 89-95.

4. Vincent MB, Luna RA, Scandiuzzi D, Novis SA. Greater occipital nerve blockade in cervicogenic headache. Arq Neuropsiquiatr 1998; 56: 720-5.

5. Bovim G, Sand T. Cervicogenic headache, migraine without aura and tension-type headache. Diagnostic blockade of greater occipital and supra-orbital nerves. Pain 1992; 51: 43-8.

6. Young W, Cook B, Malik S, Shaw J, Oshinsky M. The first 5 minutes after greater occipital nerve block. Headache 2008; 48: 1126-8.

7. Caputi CA, Firetto V. Therapeutic blockade of greater occipital and supraorbital nerves in migraine patients. Headache 1997; 37: 174-9.

8. Anthony M. Headache and the greater occipital nerve. Clin Neurol Neurosurg 1992; 94: 297-301.

9. Saadah HA, Taylor FB. Sustained headache syndrome associated with tender occipital nerve zones. Headache 1987; 27: 201-5.

10. Ambrosini A, Vandenheede M, Rossi P, Aloj F, Sauli E, Pierelli F, et al. Suboccipital injection with a mixture of rapid- and long-acting steroids in cluster headache: A double blind placebo-controlled study. Pain 2005; 118: 92-6.

11. Leinisch-Dahlke E, Jürgens T, Bogdahn U, Jakob W, May A. Greater occipital nerve block is ineffective in chronic tension type headache. Cephalalgia 2005; 25: 704-8.

12. Bartsch T, Goadsby PJ. Stimulation of the greater occipital nerve induces increased central excitability of dural afferent input. Brain 2002; 125: 1496-509.

13. Headache Classification Subcommittee of the International Headache Society. The International Classification of Headache Disorders: 2nd edition. Cephalalgia 2004; 24: 9-160.

14. Bogduk N. The anatomy and pathophysiology of neck pain. Phys Med Rehabil Clin N Am 2003; 14: 455-72.

15. Levin M. Nerve blocks in the treatment of headache. Neurotherapeutics 2010; 7: 197-203.

16. Ashkenazi A, Matro R, Shaw JW, Abbas MA, Silberstein SD. Greater occipital nerve block using local anaesthetics alone or with triamcinolone for transformed migraine: a randomised comparative study. J Neurol Neurosurg Psychiatry 2008; 79: 415-7.

17. Afridi SK, Shields KG, Bhola R, Goadsby PJ. Greater occipital nerve injection in primary headache syndromes--prolonged effects from a single injection. Pain 2006; 122: 126-9.

18. Ducic I, Moriarty M, Al-Attar A. Anatomical variations of the occipital nerves: implications for the treatment of chronic headaches. Plast Reconstr Surg 2009; 123: 859-63.

19. Schmidt D, Adelmann G. The course of the occipital artery--an anatomical investigation for biopsy in suspected vasculitis. Eur J Med Res 2001; 6: 235-41.

20. Greher M, Moriggl B, Curatolo M, Kirchmair L, Eichenberger U. Sonographic visualization and ultrasound-guided blockade of the greater occipital nerve: a comparison of two selective techniques confirmed by anatomical dissection. Br J Anaesth 2010; 104: 637-42.

21. Natsis K, Baraliakos X, Appell HJ, Tsikaras P, Gigis I, Koebke J. The course of the greater occipital nerve in the suboccipital region: a proposal for setting landmarks for local anesthesia in patients with occipital neuralgia. Clin Anat 2006; 19: 332-6.

22. Mosser SW, Guyuron B, Janis JE, Rohrich RJ. The anatomy of the greater occipital nerve: implications for the etiology of migraine headaches. Plast Reconstr Surg 2004; 113: 693-7.

23. Biondi DM. Cervicogenic headache: a review of diagnostic and treatment strategies. J Am Osteopath Assoc 2005; 105: S16-22. 